\title{
The diagnosis and molecular epidemiology investigation of avian hepatitis E in Shandong province, China
}

\author{
Kuihao Liu ${ }^{1 \dagger}$, Yiran Zhao ${ }^{1 \dagger}$, Jun Zhao ${ }^{1}$, Ningwei Geng ${ }^{1}$, Fanliang Meng ${ }^{1}$, Siqi Wang ${ }^{1}$, Jing Li ${ }^{1}$, Zhaobing Zhong ${ }^{2}$,
}

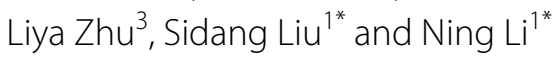

\begin{abstract}
Background: Avian hepatitis E virus (HEV) is the pathogenic agent of big liver and spleen disease (BLS) and of hepatitis-splenomegaly syndrome (HSS) in chickens, which have caused economic losses to the poultry industry in China. In this study, 18 samples of BLS chickens were collected to reveal the molecular epidemiological characteristics of avian HEV in the province of Shandong, China.

Results: Gross and microscopic lesions of clinical samples were observed; then, virology detection and genetic analysis of avian HEV were performed. The results showed that there was significant swelling and rupture in the liver and that the spleen was enlarged. Microscopic lesions demonstrated obvious hemorrhage in the liver, with infiltration of heterophilic granulocytes, lymphocytes, and macrophages, as well as the reduction of lymphocytes in the spleen. Eleven of the 18 samples were positive for avian HEV, with a positive rate of $61.11 \%$. More importantly, all avian HEV-positive samples were mixed infections: among these, the mixed infections of avian HEV and chicken infectious anemia virus (CIAV) and avian HEV and fowl adenovirus (FAdV) were the most common. Furthermore, the genetic evolution analysis showed that all avian HEV strains obtained here did not belong to the reported 4 genotypes, thus constituting a potential novel genotype.
\end{abstract}

Conclusions: These results of this study further enrich the epidemiological data on avian HEV in Shandong, prove the genetic diversity of avian HEV in China, and uncover the complex mixed infections of avian HEV clinical samples.

Keywords: Avian hepatitis E virus, Mixed infections, Potential novel genotype, Molecular epidemiology

*Correspondence: liusidang@126.com; SDTaianlining@126.com

${ }^{\dagger}$ Kuihao Liu and Yiran Zhao contributed equally to this work.

${ }^{1}$ College of Animal Science and Technology, Shandong Agricultural University, Sino-German Cooperative Research Centre for Zoonosis of Animal Origin Shandong Province, Shandong Provincial Key Laboratory of Animal Biotechnology and Disease Control and Prevention, Shandong Provincial Engineering Technology Research Center of Animal Disease Control and Prevention, 61 Daizong Road, Taian 271000, Shandong Province, China

Full list of author information is available at the end of the article

\section{Background}

Avian hepatitis E virus (HEV) can cause big liver and spleen disease (BLS) and hepatitis-splenomegaly syndrome (HSS) in chickens, which are characterized by hepatosplenomegaly. Avian HEV primarily infects laying hens and broiler breeders through fecal-oral transmission [1], resulting in decreased laying rates and increased mortality [2]. Since the first avian HEV strain was isolated and identified in China in 2010 [3], the diseases caused by avian HEV have become increasingly prevalent in chickens in recent years, causing substantial economic losses to the chicken industry in China. 
The Hepatitis E virus belong to the genus Orthohepevirus, which contains four species, designated as Orthohepevirus A, Orthohepevirus B, Orthohepevirus C, and Orthohepevirus D. Avian HEV is a non-enveloped, singlestranded, positive sense RNA virus belonging to Orthohepevirus $\mathrm{B}[4,5]$. Its genome is approximately $6.6 \mathrm{~kb}$ and contains three open reading frames (ORFs) and $3^{\prime}$ and $5^{\prime}$ non-coding regions. Of these, the capsid protein encoded by ORF2 is highly conserved and has been extensively used in viral genotyping and genetic evolution analysis of avian HEV [6].

Recently, the outbreak of hepatosplenomegaly disease has increased in chickens; although avian HEV is considered to be the major causative agent, fowl adenovirus (FAdV), reticuloendotheliosis virus (REV), avian leukosis virus (ALV), Marek's disease virus (MDV), and chicken infectious anemia virus (CIAV) can also cause hepatosplenomegaly and immunosuppression, which may facilitate the spread of avian HEV [6, 7]. Therefore, continual epidemiological investigation of avian HEV is necessary. In the present study, suspected cases of avian HEV infection were diagnosed and the molecular epidemiology of avian HEV in chickens was characterized in the province of Shandong between 2020 and 2021. These results can enhance the current understanding of the genetic diversity of avian HEV and provide new insights into prevention and control strategies of this disease.

\section{Results}

\section{Gross and microscopic lesions}

The major gross lesions of suspected cases of avian $\mathrm{HEV}$ were concentrated in the liver and spleen. In the collected samples, significant enlargement, rupture, and bleeding spots of the liver were found (Fig. 1A), and the spleen was enlarged with spots of bleeding and necrosis foci on the surface (Fig. 1B). Histopathological lesions showed hepatocellular necrosis and hemorrhagic foci in liver tissue, with massive heterophil and lymphocyte infiltration around portal areas (Fig. 2A), necrosis of liver cells and amyloid deposition with a small amount of red blood cells and macrophage infiltration (Fig. 2B), and reduced numbers of lymphocytes in the spleen and extensive amyloid deposition (Fig. 2C).

\section{Virus detection}

PCR detection revealed that 11 of the 18 samples had avian HEV infection, with a positive rate of $61.11 \%$. The 11 avian HEV-positive strains were located in the cities of Taian (2), Dezhou (2), Zibo (2), Heze (2), Linyi (1), and Jining (2), and the ages of diseased chickens were 18-25 weeks old. Interestingly, all positive samples were mixed infections, with 2 cases of avian HEV and CIAV co-infection, 1 case of avian HEV and FAdV co-infection, 5 cases of triple infection, and 3 cases of quadruple infection (Table 1). In addition, virus infections were also observed in another 5 samples; these were mixed infections of CIAV, FAdV, and MDV. No viruses were detected in the remaining 2 samples.

\section{Seque nce homology analysis and phylogenetic tree construction}

The partial ORF2 gene of the 11 avian HEV strains was amplified for sequencing and alignment. The results showed that the nucleotide homology across the 11 samples was $96.3-100 \%$, and the homology of amino acids was $96.2-100 \%$. The highest nucleotide homology was observed between the 11 avian HEV strains and others belonging to Orthohepevirus B (75.6-83.5\%). However, the homologies with Orthohepevirus A,
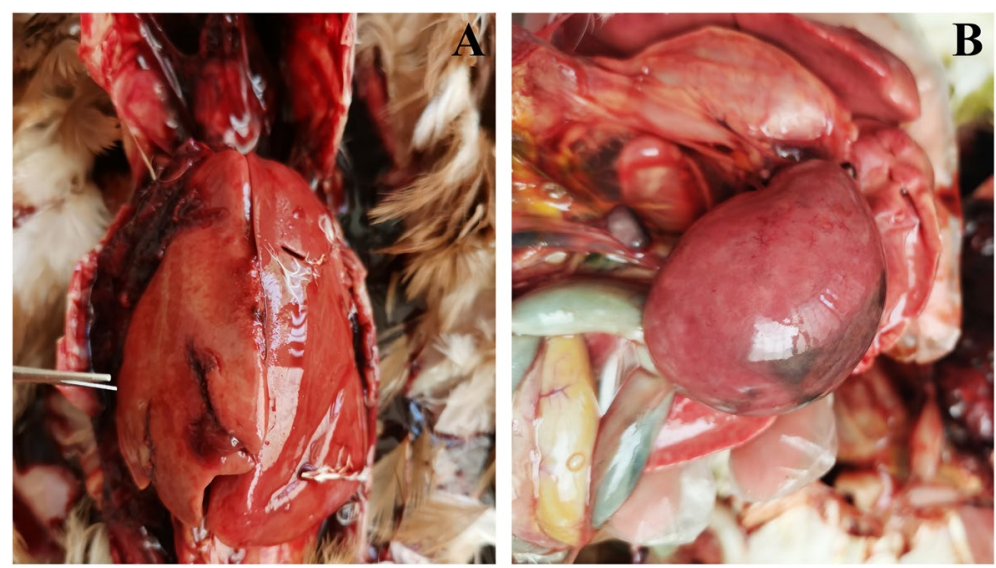

Fig. 1 Gross lesions of HEV-infected chickens. A Hemorrhage and swelling of the liver, B Splenomegaly 

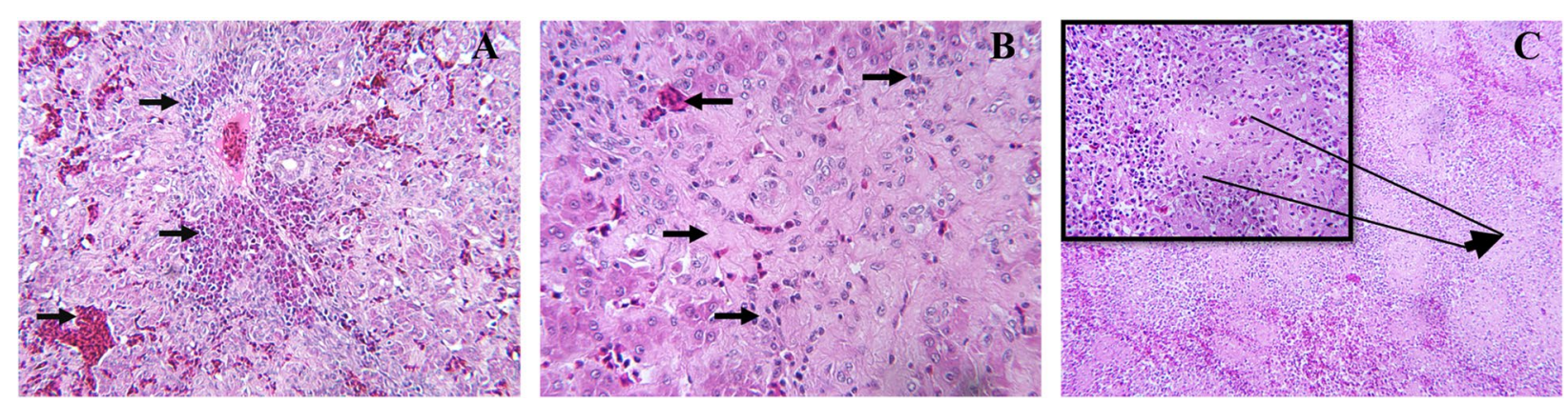

Fig. 2 Histopathological lesions of avian HEV-infected chickens. A Extensive hemorrhagic foci in liver tissue, massive infiltration of portal areas with lymphocytes and heterophils. Amplification 200x; B Degeneration and necrosis of liver cells, massive amyloid deposition, and infiltration of red blood cells and macrophage. Amplification 400x; C The decreased lymphocyte and extensive amyloid deposition in the spleen. Amplification $40 x$

Table 1 The mixed infections of avian HEV and other viruses

\begin{tabular}{lll}
\hline Type of mixed infection & Number of mixed infection cases & Viruses \\
\hline Co-infection & 2 & avian HEV, CIAV \\
Triple infection & 1 & avian HEV, FAdV \\
& 4 & avian HEV, CIAV, FAdV \\
Quadruple infection & 1 & avian HEV, CIAV, MDV \\
\hline
\end{tabular}

Orthohepevirus C, and Orthohepevirus D were low, ranging from 48.2 to $56.7 \%$. Consequently, we further analyzed the nucleotide homology between the 11 avian HEV strains and the different genotype viruses belonging to Orthohepevirus B. We found in this study that these strains had low homology with the 4 reported genotypes, ranging from 75.6 to $83.5 \%$, among which the homology with genotype 1 was the highest (78.9-83.5\%) (Table 2). The nucleotide and amino acid homologies between strains isolated in this study and potential novel genotypes that have been reported recently (accession No. MG976720, MG692744, MH094852 and MN562265) were $74.6-86.0 \%$ and $83.8-100 \%$, respectively.

The 11 avian HEV strains obtained in this study have been submitted to NCBI (accession No. MZ231098 to MZ231108), and the phylogenetic tree was constructed with other known avian HEV virus strains of different genotypes. The results demonstrate that the 11 strains did not share a branch with any known genotypes, and they constituted a single branch (Fig. 3).

\section{Discussion}

Avian HEV was first reported in 1991 in western Canada and subsequently caused outbreaks in the United States, Australia, and the United Kingdom [8, 9]. It has been reported that severe avian HEV infections have occurred in several provinces in China since 2016 [3, 10, 11]. In recent years, outbreaks of BLS and HSS in chicken flocks have gradually increased in the province Shandong [10, 12]. In order to investigate the causes of BLS and HSS along with the epidemic characteristics of avian HEV in Shandong, 18 cases of hepatomegaly and splenomegaly were collected for histopathology examination and pathogen detection, the latter including avian HEV, MDV, FAdV, ALV, CIAV, and REV. Histopathological examination revealed hemorrhage and necrosis in the liver and that the heterophagic granulocytes and lymphocytes were infiltrated in the portal areas of the liver; this was similar to the results of lymphocytic phlebitis and periphlebitis of the liver in the artificial challenge chickens infected with avian HEV $[1,13]$. Moreover, a reduction of lymphocytes and amyloidosis were observed in the spleen. However, due to the present lack of an efficient cell culture system for avian HEV isolation, the bile samples of chickens infected with avian HEV were used for animal challenge experiments, instead of purified virus [1, 13]. Therefore, the isolation of avian HEV strains and related animal experiments for the purpose of obtaining accurate results will be the focus of further research. Notably, there was a large amount of heterophagic granulocyte infiltration in the liver in this study; this type of cell is related to bacterial infection, indicating that avian HEV infections may cause secondary infections in clinical samples. The role of 
Table 2 The nucleotide and amino acid homologies between the 11 avian HEV strains and reference strains with different genotypes

\begin{tabular}{|c|c|c|c|c|c|}
\hline \multirow[t]{2}{*}{ Strains } & \multicolumn{5}{|c|}{ Different genotypes of avian $\mathrm{HEV}^{\mathrm{a}}$ (\% identity) } \\
\hline & Genotype 1 & Genotype 2 & Genotype 3 & Genotype 4 & \\
\hline \multirow[t]{2}{*}{$1-11$} & $78.9-83.5$ & $75.6-79.8$ & $81.4-83.1$ & $78.5-79.8$ & nucleotide \\
\hline & $95.0-97.5$ & $93.8-97.5$ & $96.2-98.8$ & $95.0-97.5$ & amino acid \\
\hline
\end{tabular}

a The reference viruses of the different genotypes are shown in supplementary Table 1

the mixed infections of multiple viruses in BLS and HSS cannot be ruled out.

Mixed infections of avian HEV and ALV [6] and of avian HEV and MDV have been reported since 2016 [14], and the mixed infection rate of avian HEV and several immunosuppressive viruses was up to $58 \%$ in chickens in China; co-occurring viruses were CIAV, ALV, and REV [7], indicating that the mixed infections of avian $\mathrm{HEV}$ and other viruses is common in chicken flocks. In the current study, PCR detection showed that all 11

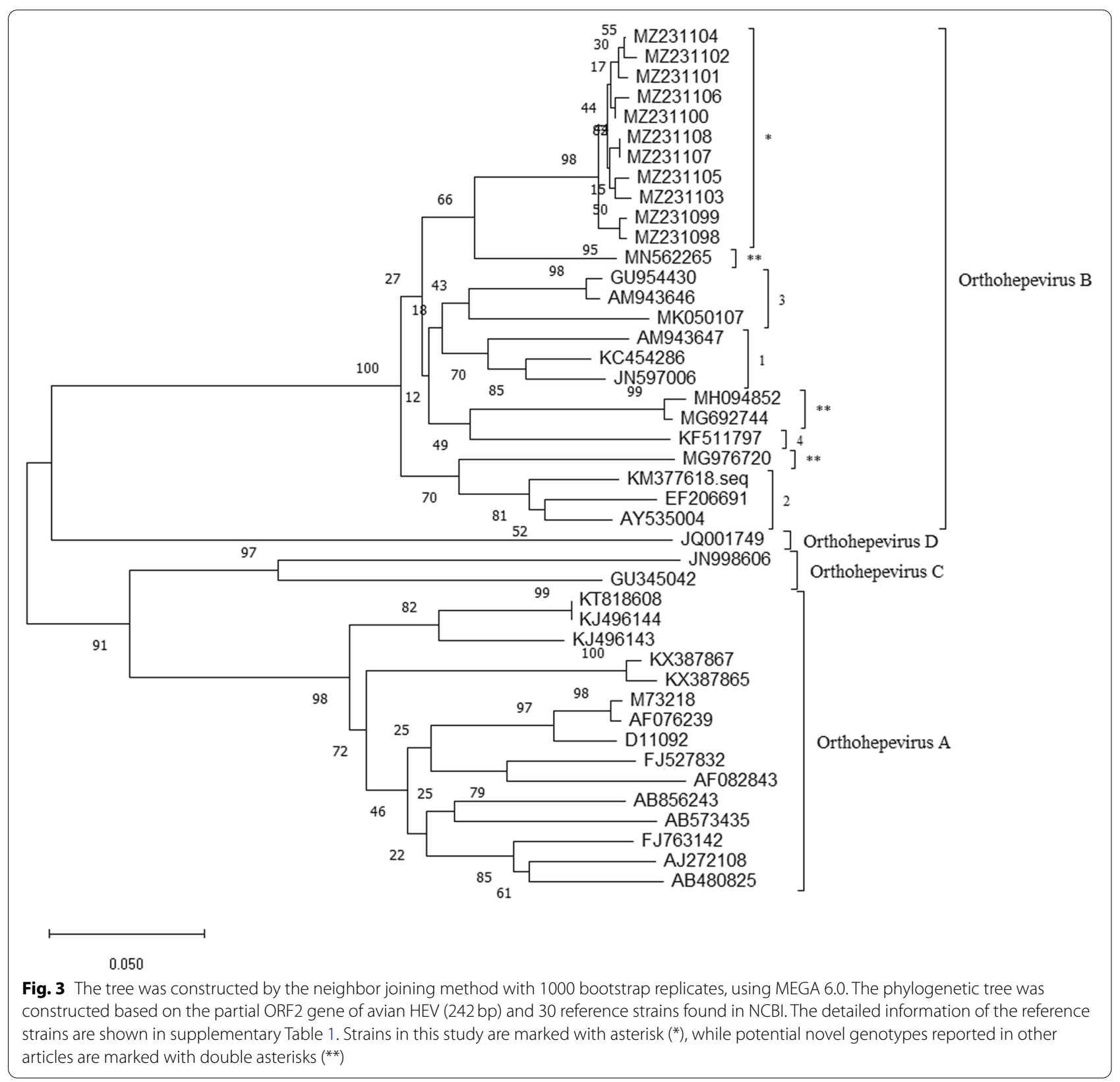


avian HEV-positive samples were mixed infections, of which the co-occurring infection rates of avian HEV and CIAV (90.90\%) and of avian HEV and FAdV (72.72\%) were highest. CIAV is a critical chicken immunosuppressive virus: according to Li et al's epidemiological survey of chicken vertical transmission or immunosuppressive virus co-infection, the detection rate of CIAV is the highest (26.5\%) [15]. Meanwhile, FAdV has been prevalent in poultry in recent years and has caused huge economic losses to the poultry industry in China $[16,17]$. It has been reported that chickens infected with FAdV were more likely to be infected with immunosuppressive diseases [18], and a previous study also reported that the coinfection rate of avian HEV and FAdV was about 10\% [7]. The problem of vaccines contaminated with CIAV and FAdV may facilitate the serious level of mixed-infection rates [19]. However, the role that CIAV and FAdV have played in the pathogenesis of avian HEV, and whether they can promote the onset of avian HEV, are questions worthy of further study.

A total of 18 samples were collected for this study, 11 of which tested positive for avian HEV and mixed infections with other viruses. It is worth noting that in two of the samples with clinical manifestations of HSS and BLS, we did not detect avian HEV or other viruses. There is still controversy regarding whether diseases characterized by HSS or BLS in chickens are completely caused by avian $\operatorname{HEV}[6,20]$. On the whole, this study confirmed the pervasiveness and severity of avian $\mathrm{HEV}$ and other viruses in chicken flocks, and continued epidemiological surveillance is required.

Different genotypes of avian HEV have been reported worldwide [10, 21-23]. Zhao et al. reported the complete genome sequence of the first avian HEV in 2010 [3]; since then, avian HEV strains of different genotypes have been reported widely in China, including in the provinces of Shandong, Jiangxi, and Guangdong [10, 13, 24]. In terms of the molecular characteristics of the 11 avian HEV strains, the nucleotide and amino acid homologies of all avian HEV strains were highest with reference strains belonging to Orthohepevirus B. Genetic evolution analysis demonstrated that all strains were located in a single branch and were different from the known 4 genotype strains, indicating a potential novel genotype. The avian HEV strains collected from different areas in Shandong had high nucleotide homology with each other, indicating that this potential novel genotype of avian HEV may be widespread in Shandong. It has been reported that avian HEV can be spread through vertical transmission [25], but further investigations are required as to whether such transmission can facilitate the spread of the potential novel genotype in different regions.
An unavoidable limitation of this study is the small number of samples. Therefore, we will expand the areas and the number of samples in our further research in order to enrich the epidemiological data.

\section{Conclusions}

In conclusion, this study found a potential novel genotype of avian HEV in Shandong, enriched the molecular epidemiological data of avian HEV, and found significant serious mixed infections of avian HEV and other viruses in clinical samples.

\section{Methods \\ Samples}

In this study, 18 clinical cases (10 dead laying hens and 8 dead broiler breeders) showing severe hepatosplenomegaly, rupture, and bleeding were collected. These samples came from different farms located in the cities of Taian, Dezhou, Zibo, Liaocheng, Heze, Linyi, Jinan, and Jining in Shandong province. The ages of affected chickens ranged from 17 to 25 weeks, and there was a significant decrease in the laying, hatching, and survival rates of chicks. The livers and spleens were collected and divided into two parts. One part of the collected tissues (about $1 \mathrm{~g}$ each) was minced and diluted 1:10 in phosphate-buffered saline (PBS, pH 7.2), then subjected to freeze-thaw cycles three times; the supernatant was collected after 12,000-rpm centrifugation for $5 \mathrm{~min}$ and stored at $-80^{\circ} \mathrm{C}$ for virus detection. The other part of the tissues was fixed with $10 \%$ formalin solution, routinely processed, and stained using the HE method for histopathological examination [26].

\section{Nucleic acid extraction and virus detection}

Each sample's liver and spleen were mixed and ground to extract nucleic acid. The nucleic acid (RNA/DNA) was extracted from the treated samples using the Simply P Virus DNA/RNA Extraction Kit (Bioer, Hangzhou, China), according to the procedures described in the manufacturer's instructions. The obtained virus nucleic acids were divided into two parts: one part was reversetranscribed into cDNA by ReverTra Ace qPCR RT Kit (TOYOBO, Shanghai, China) for the detection of RNA viruses-including avian HEV, ALV, and REV-and another part was used for the detection of DNA viruses (MDV, FAdV, and CIAV). Virus detection was conducted by the PCR method. All primers refer to previous research $[16,20]$ (Table 3). In line with the instructions of $2 \times$ Accurate Taq Master Mix (Dye Plus) (Accurate Biotechnology, Hunan, China), the total volume of the reaction system was $20 \mu \mathrm{L}$, and the thermal cycling conditions 
Table 3 The primers used in the study

\begin{tabular}{|c|c|c|c|}
\hline Primers & Sequence $\left(5^{\prime}-3^{\prime}\right)$ & Product size (bp) & Annealing temperature $\left({ }^{\circ} \mathrm{C}\right)$ \\
\hline CIAV-F & CAGAATTCCCACCTCAAGCGACTTCGAC & 580 & 54 \\
\hline CIAV-R & ATGTCGACGGGGCTGAAGGAT & & \\
\hline REV-F & CATACTGAGCCAATGGTT & 300 & 54 \\
\hline REV-R & AATGTTGTAGCGAAGTACT & & \\
\hline MDV-F & TCATCAGGGTCTCCCGTCACCT & 1005 & 55 \\
\hline MDV-R & AGAGATGTCTCAGGAGCCAGAG & & \\
\hline FAdV-F & AATTTCGACCCCATGACGCGCCAGG & 508 & 56 \\
\hline FAdV-R & TGGCGAAAGGCGTACGGAAGTAAGC & & \\
\hline ALV-J-F & GGATGAGGTGACTAAGA & 512 & 56 \\
\hline ALV-J-R & CGAACCAAAGGTAACACACG & & \\
\hline Avian HEV-F1 & TCGCCT(C)GGTAAT(C)ACA(T)AATGC & 278 & 60 \\
\hline Avian HEV-R1 & GCGTTC(G)CCG(C)ACAGGT(C)CGGCC & & \\
\hline Avian HEV-F2 & ACA(T)AATGCT(C)AGGGTCACCCG & 242 & 56 \\
\hline Avian HEV-R2 & ATGTACTGA(G)CCA(G)CTG(C)GCCGC & & \\
\hline
\end{tabular}

were as follows: $94^{\circ} \mathrm{C}$ for $30 \mathrm{~s}, 35$ cycles of $98^{\circ} \mathrm{C}$ for $10 \mathrm{~s}$, $54-60^{\circ} \mathrm{C}$ for $30 \mathrm{~s}$, and $72^{\circ} \mathrm{C}$ for $1 \mathrm{~min}$, with a final incubation at $72^{\circ} \mathrm{C}$ for $2 \mathrm{~min}$.

\section{Phylogenetic analysis of avian HEV strains}

The PCR-positive products were cloned to the pMD18-T vector (Takara, Dalian, China) and were sent to Sangon Biotech (Shanghai, China) for sequencing. Sequences of avian HEV strains generated in this study were submitted to the GenBank under accession numbers MZ231098MZ231108. The phylogenetic and molecular evolutions of these avian HEV strains were analyzed using MEGA version 6 [27] by the neighbor joining method with 1000 bootstrap replicates, and the comparison of sequence identities was performed using MegAlign software (DNAStar, Madison, United States).

\section{Abbreviations}

PCR: Polymerase chain reaction; PBS: Phosphate-buffered saline; Avian HEV: Avian hepatitis E virus; BLS: Big liver and spleen disease; HSS: Hepatitis-splenomegaly syndrome; FAdV: Fowl adenovirus; REV: Reticuloendotheliosis virus: ALV: Avian leukosis virus; MDV: Marek's disease virus; CIAV: Chicken infectious anemia virus; HE: Hematoxylin-eosin staining.

\section{Supplementary Information}

The online version contains supplementary material available at https://doi. org/10.1186/s12917-021-03079-2.

Additional file 1 Supplementary Table 1. The information of reference strains in the study.

\section{Acknowledgments}

We thank all the anonymous reviewers from the journal for their helpful comments. We also thank all researchers who shared avian HEV genome sequences in GenBank.

\section{Authors' contributions}

$\mathrm{KL}$ and $\mathrm{YZ}$ performed the experiment and wrote the manuscript, JZ, NG, FM, SW, JL, LZ and ZZ collected and analyzed data, SL and NL conceived the study and reviewed the manuscript. All authors have read and approved the manuscript.

\section{Funding}

This research was supported by National Key Research and Development Program (Grants No. 2016YFD0500106), Funds of Shandong "Double Tops" Program, China Postdoctoral Science Foundation Funded Project (2019 M652450), High-level Scientific Research Foundation for the introduction of talent of Shandong Agricultural University. These four programs supported entirely this project starting from the design of study, sample collection, analysis and interpretation of data.

\section{Availability of data and materials}

The viral sequences obtained in this study are deposited in GenBank under the accession numbers: MZ231098 to MZ231108. The data involving in the manuscript can be obtained from the corresponding author upon reasonable request.

\section{Declarations}

\section{Ethics approval and consent to participate}

This study was approved by the Research Ethics Committee of Shandong Agricultural University. There were no vulnerable populations involved, and no endangered species were used in the experiments. Farm managers gave permission for their animal samples to be used in this study.

\section{Consent for publication}

Not applicable.

\section{Competing interests}

The authors declare that they have no competing interests.

\section{Author details}

${ }^{1}$ College of Animal Science and Technology, Shandong Agricultural University, Sino-German Cooperative Research Centre for Zoonosis of Animal Origin Shandong Province, Shandong Provincial Key Laboratory of Animal Biotechnology and Disease Control and Prevention, Shandong Provincial Engineering Technology Research Center of Animal Disease Control and Prevention, 61 Daizong Road, Taian 271000, Shandong Province, China. ${ }^{2}$ Taian Daiyue District Administrative Examination and Approval Service Bureau, Taian 271018, Shandong Province, China. ${ }^{3}$ Animal Husbandry and Veterinary Service Centre of Linshu, Linyi 276700, Shandong Province, China. 
Received: 14 June 2021 Accepted: 16 November 2021

Published online: 25 January 2022

\section{References}

1. Liu B, Sun Y, Chen Y, Du T, Nan Y, Wang X, et al. Effect of housing arrangement on fecal-oral transmission of avian hepatitis $E$ virus in chicken flocks. BMC Vet Res. 2017;13(1):282.

2. Hsu IW, Tsai HJ. Avian hepatitis E virus in chickens, Taiwan, 2013. Emerg Infect Dis. 2014;20(1):149-51.

3. Zhao Q, Zhou EM, Dong SW, Qiu HK, Zhang L, Hu SB, et al. Analysis of avian hepatitis E virus from chickens, China. Emerg Infect Dis. 2010;16(9):1469-72.

4. Sridhar S, Teng JLL, Chiu TH, Lau SKP, Woo PCY. Hepatitis E virus genotypes and evolution: emergence of camel hepatitis E variants. Int J Mol Sci. 2017;18(4):869.

5. Doceul V, Bagdassarian E, Demange A, Pavio N. Zoonotic hepatitis E virus: Classi fi cation, Animal Reservoirs and Transmission Routes. Viruses. 2016:8(10):270

6. Sun YN, Lu QZ, Zhang JF, Li XX, Zhao JK, Fan WQ, et al. Co-infection with avian hepatitis $\mathrm{E}$ virus and avian leukosis virus subgroup $\mathrm{J}$ as the cause of an outbreak of hepatitis and liver hemorrhagic syndromes in a brown layer chicken flock in China. Poultry Sci. 2020:99(3):1287-96.

7. Su Q, Zhang Y, Li Y, Cui Z, Chang S, Zhao P. Epidemiological investigation of the novel genotype avian hepatitis E virus and co-infected immunosuppressive viruses in farms with hepatic rupture haemorrhage syndrome, recently emerged in China. Transbound Emerg Dis. 2019;66(2):776-84.

8. Bilic l, Jaskulska B, Basic A, Morrow CJ, Hess M. Sequence analysis and comparison of avian hepatitis $E$ viruses from Australia and Europe indicate the existence of different genotypes. J Gen Virol. 2009;90(Pt 4):863-73.

9. Primadharsini PP, Nagashima S, Okamoto H. Genetic variability and evolution of hepatitis E virus. Viruses. 2019;11(5):456.

10. Su Q, Liu Y, Cui Z, Chang S, Zhao P. Genetic diversity of avian hepatitis E virus in China, 2018-2019. Transbound Emerg Dis. 2020;67(6):2403-7.

11. Li H, Zhang F, Tan M, Zeng Y, Yang Q, Tan J, et al. Research note: a putative novel subtype of the avian hepatitis E virus of genotype 3, Jiangxi province, China. Poult Sci. 2020;99(12):6657-63.

12. Yu G, Lin Y, Dou Y, Tang Y, Diao Y. Prevalence of fowl adenovirus serotype 4 and co-infection by immunosuppressive viruses in fowl with Hydropericardium hepatitis syndrome in Shandong Province, China. Viruses. 2019;11(6):517.

13. Su Q, Li Y, Meng F, Cui Z, Chang S, Zhao P. Hepatic rupture hemorrhage syndrome in chickens caused by a novel genotype avian hepatitis $E$ virus. Vet Microbiol. 2018:222:91-7.

14. Yang S, Wang L, Sun S. Natural infection with avian hepatitis E virus and Marek's disease virus in Brown layer chickens in China. Avian Dis. 2016;60(3):698-704.

15. Li X, Zhang KR, Pei Y, Xue J, Ruan SF, Zhang GZ. Development and application of an MRT-qPCR assay for detecting Coinfection of six vertically transmitted or immunosuppressive avian viruses. Front Microbiol. 2020;1 1:1581

16. Niu YJ, Sun W, Zhang GH, Qu YJ, Wang PF, Sun HL, et al. Hydropericardium syndrome outbreak caused by fowl adenovirus serotype 4 in China in 2015. J Gen Virol. 2016;97(10):2684-90.

17. Niu Y, Sun Q, Shi Y, Ding Y, Li Z, Sun Y, et al. Immunosuppressive potential of fowl adenovirus serotype 4. Poult Sci. 2019;98(9):3514-22.

18. Meng F, Dong G, Zhang Y, Tian S, Cui Z, Chang S, et al. Co-infection of fowl adenovirus with different immunosuppressive viruses in a chicken flock. Poult Sci. 2018;97(5):1699-705.

19. Su Q, Meng F, Li Y, Zhang Y, Zhang Z, Cui Z, et al. Chicken infectious anemia virus helps fowl adenovirus break the protection of maternal antibody and cause inclusion body hepatitis-hydropericardium syndrome in layers after using co-contaminated Newcastle disease virus-attenuated vaccine. Poultry Sci. 2019;98(2):621-8.

20. Sun ZF, Larsen CT, Dunlop A, Huang FF, Pierson FW, Toth TE, et al. Genetic identification of avian hepatitis $E$ virus (HEV) from healthy chicken flocks and characterization of the capsid gene of 14 avian HEV isolates from chickens with hepatitis-splenomegaly syndrome in different geographical regions of the United States. J Gen Virol. 2004;85(Pt 3):693-700.
21. Sun $P$, Lin $S, H$ He, Zhou EM, Zhao Q. Avian hepatitis E virus: with the trend of genotypes and host expansion. Front Microbiol. 2019;10:1696.

22. Iqbal T, Rashid U, Idrees M, Afroz A, Kamili S, Purdy MA. A novel avian isolate of hepatitis E virus from Pakistan. Virol J. 2019;16(1):142.

23. Kwon HM, Sung HW, Meng XJ. Serological prevalence, genetic identification, and characterization of the first strains of avian hepatitis $E$ virus from chickens in Korea. Virus Genes. 2012:45(2):237-45.

24. Sun Y, Du T, Liu B, Syed SF, Chen Y, Li H, et al. Seroprevalence of avian hepatitis $E$ virus and avian leucosis virus subgroup $\mathrm{J}$ in chicken flocks with hepatitis syndrome, China. BMC Vet Res. 2016;12(1):261.

25. Troxler S, Pac K, Prokofieva I, Liebhart D, Chodakowska B, Furmanek D, et al. Subclinical circulation of avian hepatitis $\mathrm{E}$ virus within a multipleage rearing and broiler breeder farm indicates persistence and vertical transmission of the virus. Avian Pathol. 2014:43(4):310-8.

26. Cardiff RD, Miller CH, Munn RJ. Manual hematoxylin and eosin staining of mouse tissue sections. Cold Spring Harb Protoc. 2014;2014(6):655-8.

27. Tamura K, Stecher G, Peterson D, Filipski A, Kumar S. MEGA6: molecular evolutionary genetics analysis version 6.0. Mol Biol Evol. 2013;30(12):2725-9.

\section{Publisher's Note}

Springer Nature remains neutral with regard to jurisdictional claims in published maps and institutional affiliations.
Ready to submit your research? Choose BMC and benefit from:

- fast, convenient online submission

- thorough peer review by experienced researchers in your field

- rapid publication on acceptance

- support for research data, including large and complex data types

- gold Open Access which fosters wider collaboration and increased citations

- maximum visibility for your research: over $100 \mathrm{M}$ website views per year

At BMC, research is always in progress.

Learn more biomedcentral.com/submissions 